\title{
Publisher Correction: TDP-43 gains function due to perturbed autoregulation in a Tardbp knock-in mouse model of ALS-FTD
}

Matthew A. White, Eosu Kim, Amanda Duffy, Robert Adalbert, Benjamin U. Phillips, Owen M. Peters, Jodie Stephenson, Sujeong Yang, Francesca Massenzio, Ziqiang Lin, Simon Andrews, Anne Segonds-Pichon, Jake Metterville, Lisa M. Saksida, Richard Mead, Richard R. Ribchester, Youssef Barhomi, Thomas Serre, Michael P. Coleman, Justin R. Fallon, Timothy J. Bussey, Robert H. Brown Jr and Jemeen Sreedharan (1)

Correction to: Nature Neuroscience https://doi.org/10.1038/s41593-018-0113-5, published online 19 March 2018.

In the version of this article initially published, the footnote number 17 was missing from the author list for the two authors who contributed equally. Also, the authors have added a middle initial for author Justin R. Fallon and an acknowledgement to the Babraham Institute Imaging Facility and Sequencing Core Facility. The errors have been corrected in the HTML and PDF versions of the article.

Published online: 5 June 2018

https://doi.org/10.1038/s41593-018-0160-y

\section{Publisher Correction: Thalamic projections sustain prefrontal activity during working memory maintenance}

Scott S Bolkan, Joseph M Stujenske, Sebastien Parnaudeau, Timothy J Spellman, Caroline Rauffenbart, Atheir I Abbas, Alexander Z Harris, Joshua A Gordon and Christoph Kellendonk

Correction to: Nature Neuroscience https://doi.org/10.1038/nn.4568, published online 3 May 2017.

In the version of this article initially published, the title of ref. 45 was given as "Sustaining cortical representations by a content-free thalamic amplifier." The correct title is "Thalamic amplification of cortical connectivity sustains attentional control." The error has been corrected in the HTML and PDF versions of the article.

Published online: 31 May 2018

https://doi.org/10.1038/s41593-018-0132-2

\section{Publisher Correction: Learning by neural reassociation}

Matthew D. Golub (D), Patrick T. Sadtler, Emily R. Oby, Kristin M. Quick, Stephen I. Ryu, Elizabeth C. Tyler-Kabara (D), Aaron P. Batista, Steven M. Chase (iD and Byron M. Yu (D)

Correction to: Nature Neuroscience https://doi.org/10.1038/s41593-018-0095-3, published online 20 March 2018.

In the version of this article initially published, equation (10) contained $\cos \Theta$ instead of sin $\Theta$ as the bottom element of the right-hand vector. The error has been corrected in the HTML and PDF versions of the article. 